\title{
Datenmigration aus Altsystemen nach SAP/4HANA
}

\begin{abstract}
Matthias Förg
Im Jahr 2027 endet der Hersteller-Support für klassische SAP ERP-Lösungen. Gegen einen Aufpreis ist ein Support jedoch noch drei weitere Jahre möglich. Ab 2030 wird dann endgültig nur noch SAP S/4HANA unterstützt. Deshalb besteht für Unternehmen, die den Wechsel auf die neue Technologie noch nicht geplant haben, derzeit akuter Handlungsbedarf. Viele Unternehmen haben dabei großen Respekt vor der Komplexität einer solchen Migration, insbesondere im Hinblick auf die Stammdaten. Eine Herausforderung, die Unternehmen jedoch mit sorgfältiger Vorbereitung und der passenden Strategie gut meistern können. Dieser Beitrag liefert Tipps für eine erfolgreiche Migration.
\end{abstract}

Eine unternehmensweite Datenmigration ist aufgrund des veränderten Datenmodells von SAP S/4HANA bei einem Systemwechsel zwingend nötig. Es scheint allerdings so, als hätten einige Unternehmen diese Dringlichkeit noch nicht erkannt: So haben von 122 IT-Verantwortlichen aus unterschiedlichen Branchen, die bei einer Umfrage [1] interviewt wurden, $22 \%$ noch gar keine Entscheidung hinsichtlich einer Systemablösung getroffen, ein Drittel bereitet gerade die Migration vor - und das, während SAP im Hintergrund bereits die Tage zählt. Dafür ist anderen Unternehmen die Dringlichkeit umso bewusster. Einer Umfrage der Beratungsgesellschaft Lünendonk [2] in Zusammenarbeit mit KPMG und Rödl \& Partner aus dem Herbst 2020 zufolge gibt ein Viertel der befragten Entscheidungsträger den von SAP ausgeübten Zeitdruck als Wechselgrund an. Doch ist die Migration auf SAP S/4HANA auch mit positiven Effekten verbunden.

Eine Stärke der neuen Technologie ist ihre Geschwindigkeit. Durch das hohe Verarbeitungs- und Reaktionstempo können beispielsweise Materialfehlbestände oder Maschinenausfälle schneller erkannt und kompensiert werden als in herkömmlichen ERP-Umgebungen. Da das System auf der In-Memory-Datenbank SAP HANA basiert,

In diesem Beitrag lesen Sie:

- welche Rolle Daten bei der Migration nach SAP S/4HANA und bei der Wahl der Migrationsstrategie spielen,

- wie die Datenqualität die Migration beeinflusst und welche Chancen das Projekt bietet,

- wie Unternehmen die Datenqualität verbessern und kontinuierlich hochhalten können. ist es zudem in der Lage, Simulationen, Transaktionen, Prognosen und Empfehlungen in Echtzeit abzuwickeln. Dies sorgt nicht nur für schnelle Reaktionsmöglichkeiten im operativen Geschäft, sondern auch für verbesserte Prozesse und damit für geringere Ausfallkosten und eine höhere Produktqualität. Ein weiterer Pluspunkt ist der deutlich geringere Speicher- und damit Hardwarebedarf, da SAP S/4HANA Daten nicht wie üblich über eine Festplatte speichert, sondern diese im Arbeitsspeicher sichert. Auch an der Bedienoberfläche und dem Rollen- und Rechtesystem hat SAP einiges verbessert. Zum einen läuft die Benutzeroberfläche auf allen mobilen Endgeräten einwandfrei und erlaubt so einen deutlich flexibleren Einsatz als herkömmliche ERP-Systeme. Außerdem erhalten Mitarbeiterinnen und Mitarbeiter nur noch Zugriff auf Daten und Anwendungen, die für sie tatsächlich relevant sind.

\section{Das Datenmodell von SAP S/4HANA}

Neben diesen Vorteilen von SAP S/4HANA gegenüber alten ERP-Systemen zeichnet sich die neue Lösung vor allem durch ihr komplett überarbeitetes Datenmodell aus. Das Grundprinzip des neuen Ansatzes ist Einfachheit. Die Verschlankung der Datenbankstruktur zielt im Wesentlichen darauf ab, Geschäftsprozesse zu verbessern. Zur Veranschaulichung: Herkömmliche ERP-Systeme unterscheiden noch zwischen den finanzbuchhalterischen Entitäten „Debitor" und "Kreditor" sowie einem neutralen "Geschäftspartner". Jede der drei Kategorien besitzt zahlreiche Eigenschaften wie Personen- und Adressdaten, Buchungskonten und Bankverbindungen oder auch Rollenbezeichnungen für die Personen, um nur einige zu nennen. 


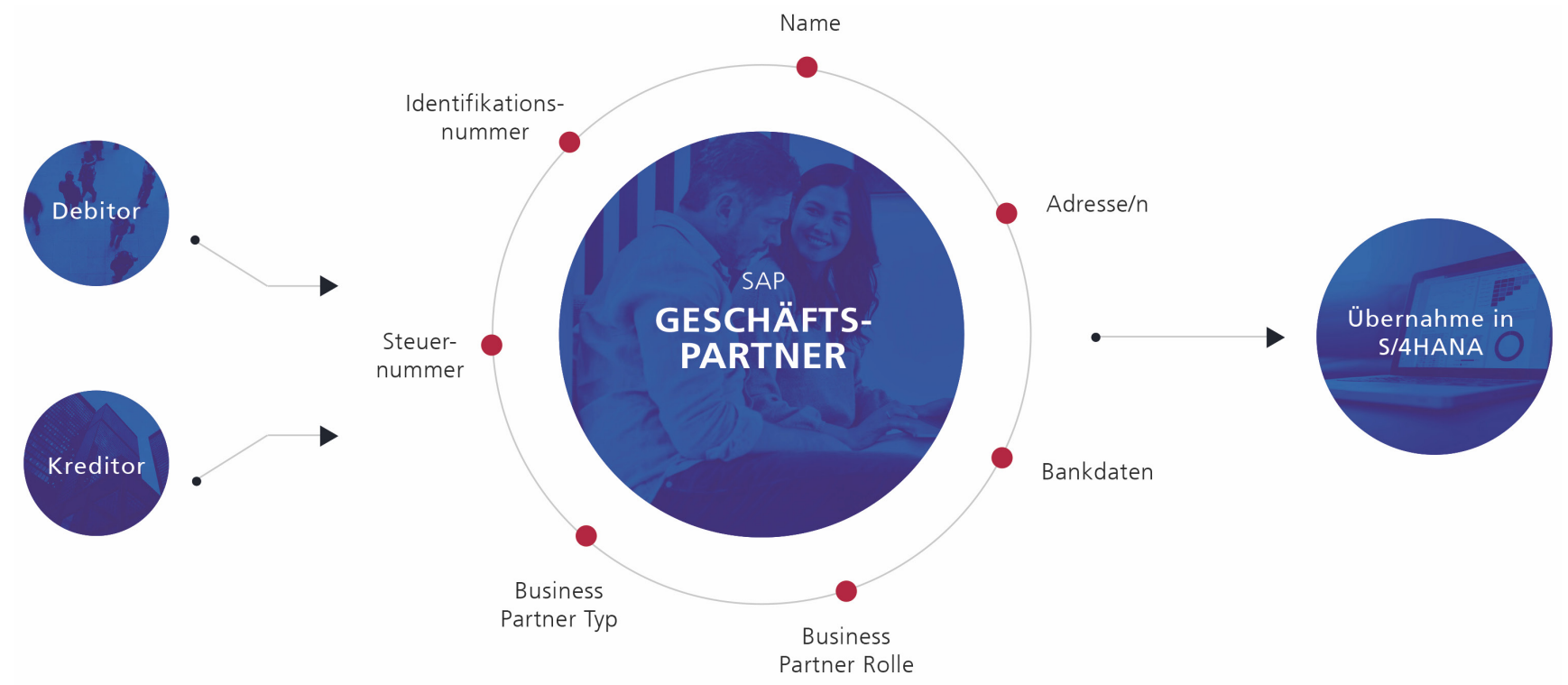

UNISERV DATA QUALITY

Bild 1: Das neue Datenmodell von SAP S4/HANA vereint Entitäten wie Debitor und Kreditor zu einem einheitlichen Geschäftspartnerdatenstamm.

Im neuen Datenbanksystem von SAP S/4HANA wird nur ein einzelner, einheitlicher Geschäftspartnerstamm angelegt, der die alten Entitäten in sich vereint und die Unterscheidung der Rolle, der geographischen Lokalität und anderen Systematiken auf einer neuen Abstraktionsebene als Metainformationen abbildet (Bild 1). Außerdem entfallen fehleranfällige Workflows, wie etwa der Datenabgleich zwischen der Finanzbuchhaltung und dem Controlling während des Jahresabschlusses. Damit werden zugleich besonders mühsame und störanfällige Abläufe minimiert. Auch für andere Segmente, wie etwa die Projektplanung im Bausektor, bietet der Einsatz von SAP S/4HANA eine Modernisierung und damit effizientere Abwicklung von Prozessen. Echtzeitdaten schaffen eine transparente Sicht auf Liefer- und Beschaffungsketten und unterstützen damit Planungsabläufe am Bau. Es gibt zahlreiche ähnliche Beispiele dafür, wie Unternehmen mit SAP S/4HANA Effizienz und Produktivität steigern können. Geschenkt gibt es diesen Bonus allerdings nicht: Eine Eins-zu-eins-Migration aus Bestandssystemen und den bisherigen SAP-Lösungen ist nicht immer sinnvoll. Vielmehr wird eine Migrationsstrategie benötigt, die zum Unternehmen, seinen Geschäftsprozessen, seiner Softwarearchitektur und seiner Datenhaltung passt.

\section{Strategie}

Die Migration von Stammdaten stellt eine große Herausforderung dar. Die Menge der Datensätze je nach Unternehmenstyp und -größe kann beachtlich sein. Zu- dem sind sie die Basis für einen Großteil der Geschäftsprozesse. Es muss also sichergestellt werden, dass die Datenmigration fehlerfrei und vollständig erfolgt - und Strukturen abbildet, die bis hinein in die operativen Ebenen tragfähig sind. Aus diesem Blickwinkel wird deutlich, dass die Migration zu SAP S/4HANA zugleich eine enorme Chance bietet: Unternehmen können ihre Datenqualität

Bild 2: Laut Uniserv-Studie wählen je ein Drittel der befragten Unternehmen den "Greenfield"- oder den „Brownfield"Migrationsansatz.

\section{Migrations-Ansatz}

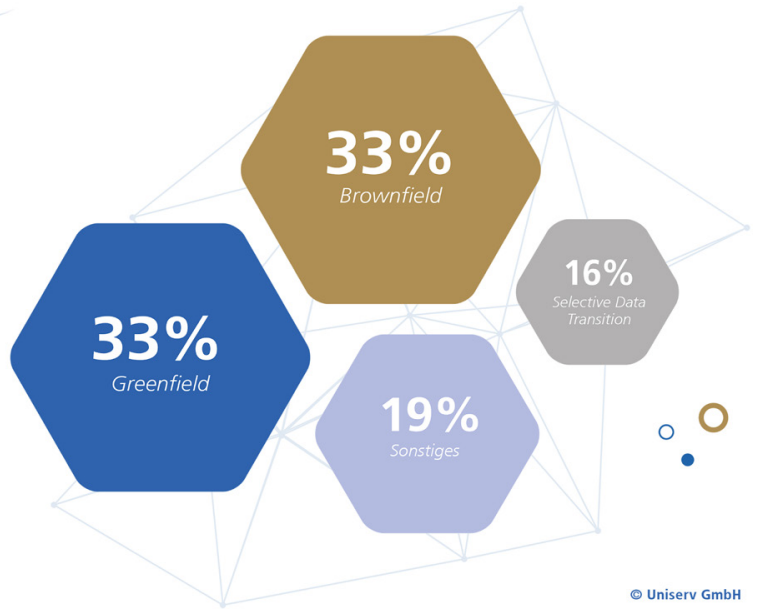


Bild 3: Unternehmen kämpfen bei der Datenqualität vor allem mit den Problemen, die veraltete, unvollständige Daten und Dubletten sowie Datensilos verursachen.
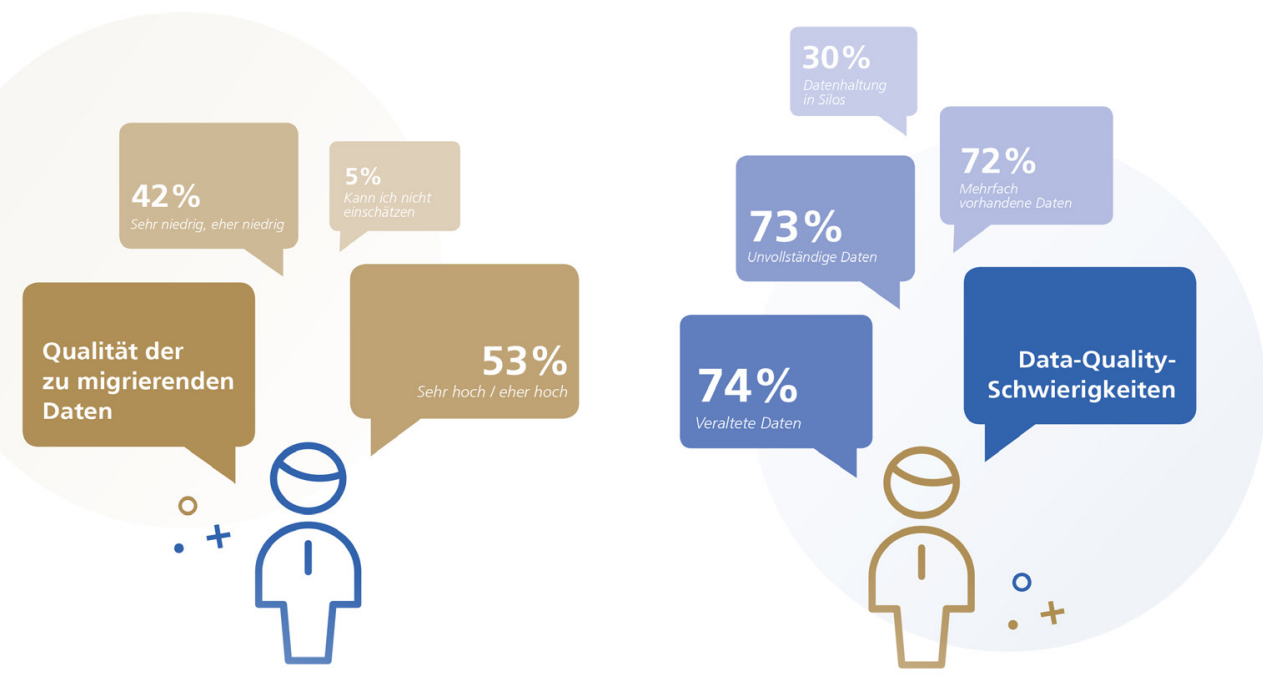

verbessern und Prozesse schlanker gestalten. Schließlich zeigt sich ein erfolgreicher Systemwechsel nicht nur darin, wie effizient mit der neuen Technologie gearbeitet wird, sondern auch dadurch, wie sich die neue Anwendungslandschaft auf der Prozessebene und damit im operativen und strategischen Geschäft auswirkt. Damit die Stärken von SAP S/4HANA zur Geltung kommen, sollten Unternehmen eine Bestandsaufnahme durchführen, bevor sie in die tiefere Planung gehen. Fragen, die während einer IST-Analyse nicht vergessen werden sollten, lauten zum Beispiel: Welche Release-Version läuft aktuell, welche Support Packages sind im Einsatz? Welches Datenbanksystem wird genutzt? Welche Quell- und Zielsysteme werden betrieben? Ist das Altsystem in Non-Unicode codiert, läuft es auf Dual Stack? Wie lang darf die maximale Downtime sein? All diese Fragestellungen entscheiden darüber, wie aufwendig die Migration wird - und sein darf.

\section{„Brownfield“, „,Greenfield“ oder „Bluefield“"}

Derzeit existieren zwei Ansätze, die von Unternehmen zu gleichen Teilen favorisiert werden: $33 \%$ der Unternehmen setzen laut Uniserv-Umfrage auf den sogenannten „Brownfield"-Ansatz, ein weiteres Drittel auf den "Greenfield"-Ansatz (Bild 2). Der wesentliche Unterschied dieser beiden Herangehensweisen liegt darin, dass die eine Methode eher einer Systemkonvertierung mit Software-Upgrade gleicht, während die zweite für eine komplett neue Implementierung ",auf der grünen Wiese" steht. Anwender der klassischen SAP-Systeme R/3, Business Suite und ERP 6.0 ohne weitere Drittsysteme oder Legacy-Datenbanken entscheiden sich häufig für den „Brownfield“-Ansatz: Hier wird das bisherige System mit der kompletten Systemlandschaft auf und nach SAP S/4HANA migriert. Ein Nachteil dieses Vorgehens liegt in der Gefahr, unnötige Altlasten mit in die neue Systemlandschaft um- zuziehen. Wer das vermeiden möchte, setzt alles auf neu: Der "Greenfield"-Ansatz hat den bestechenden Vorteil, dass nur die Daten migriert werden, die künftig wirklich noch gebraucht werden. So lässt sich unter anderem die Leistungsfähigkeit der In-Memory-Datenbank besser ausschöpfen. Eine weitere Möglichkeit, die laut der Befragung jedoch nur von $16 \%$ der Unternehmen genutzt wird, ist die selektive Migration - auch Selective Data Transition oder "Bluefield“-Methode genannt. Sie stellt einen Mittelweg aus "Greenfield" und "Brownfield" dar. Hierbei handelt es sich um einen Strategieansatz, bei dem das alte System ohne Daten kopiert und auf SAP S/4HANA umgestellt wird. Im Anschluss wird dieses leere System per Datenmigration gezielt mit Altdaten gefüllt. Eine Stärke dieser Vorgehensweise ist die Individualität, mit der sie gesteuert werden kann. Die Migrationsbeauftragten migrieren zum Beispiel nur einen definierten Ausschnitt von Daten oder schlüsseln Daten um.

\section{Datenqualität entscheidet über Migrationserfolg}

Steht die für ein Unternehmen und seine IT-Landschaft passende Wechselstrategie fest, muss die Datenqualität sichergestellt werden. Dieser zentrale Schritt muss in jedem Fall, egal ob „Brown"-, „Green"- oder „Bluefield", mit aller Sorgfalt vollzogen werden. Schließlich ist die Qualität der Quelldaten entscheidend für den Gesamterfolg der SAP S/4HANA-Migration. Sind die Ausgangsdaten überholt, unvollständig oder voller Dubletten, kann das neue System niemals sein volles Potenzial zur Modernisierung der digitalen Geschäftsprozesse entfalten.

Neben veralteten Daten sind vor allem doppelte und mehrfach vorhandene Daten die größte Herausforderung in Sachen Datenqualität: Der Uniserv-Umfrage zufolge konnten $83 \%$ der Unternehmen, welche die SAP S/4HANA-Migration 
bereits abgeschlossen haben, veraltete, unvollständige Daten und Dubletten sowie Datensilos als Hauptproblem ausmachen (Bild 3). Angesichts der Fülle unterschiedlicher Strukturen und Formate, in denen Unternehmensdaten vorgehalten werden können, ist die Sicherung der Datenqualität gerade im globalen Geschäftsverkehr keine banale Sache. Oft sind die Datenquellen über verschiedene Silos unternehmensweit verteilt - nicht nur über Standorte, sondern zum Teil auch über Kontinente hinweg. Aus diesem Grund lohnt sich für Unternehmen besonders bei globalen Datenmigrationsprojekten der Einsatz professioneller Tools, die beispielsweise die automatisierte Massenprüfung von Geschäftspartnerdaten auf postalische Korrektheit, die Bereinigung von Dubletten und die Vervollständigung von Datensätzen per Identity Resolution übernehmen können.

\section{Kurzfristiger Aufwand, langfristige Wirkung}

SAP S/4HANA setzt sehr hohe Qualitätsnormen für Stammdaten und deren Einhaltung durch eine Reihe von Prüfregeln durch. Technische Inkonsistenzen, die bei diesen Prüfungen vor der Konvertierung festgestellt wurden, müssen unabhängig vom Übergangsszenario behoben werden. Deshalb ist es wichtig, dass die Stammdaten schon vor dem Projektstart und nicht erst im laufenden Projekt eingearbeitet werden. Die Konsolidierung von Daten aus unterschiedlichen Quellsystemen und ihre Zusam-

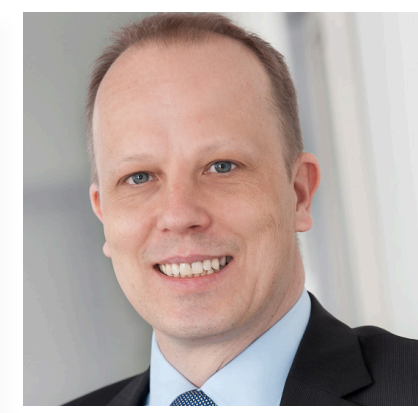

Matthias Förg ist Head of Sales bei Uniserv und verantwortet den weltweiten Vertrieb der Uniserv-Lösungen für Datenqualität und Kundendatenmanagement.

Kontakt:

Matthias Förg

Uniserv GmbH

Rastatter Straße 13

75179 Pforzheim

www.uniserv.com menführung zu einer 360-Grad-Sicht auf den Geschäftspartner als "Golden Record" verschafft Unternehmen eine einheitliche Sicht auf ihre Kunden und Geschäftspartner. Dahinter verbirgt sich zunächst ein gewisser Initialaufwand. Wenn jedoch Unternehmen eine übergreifende, konsolidierte Sicht auf Ressourcen, Kunden und Partner haben, können sie ihre Wertschöpfungsketten mit maximaler Effizienz aussteuern. Die Migration zu SAP S/4HANA kann mit einer guten Vorbereitung und der passenden Strategie einen soliden Teil dazu beitragen. Deshalb sind Unternehmen gut beraten, schon heute mit dem Wechsel auf die neue SAP-Umgebung zu beginnen.

\section{Literatur:}

[1] Uniserv stellt Ergebnisse der Praxisstudie S/4HANA-Migration: Fokus Stammdatenmigration vor. (22.10.2020). Abgerufen am 21.06.2021, von https://www.uniserv.com/lp-presse-studie-s4-hana/

[2] S/4HANA-Umstellung: Status quo, Planungen und Roadmap zur ERP-Modernisierung in der Corona-Pandemie. (11.03.2021). Abgerufen am 21.06.2021, von https://www.luenendonk.de/produkte/ studien-publikationen/luenendonk-studie-2021-s4hana-umstellung-status-quo-planungen-und-roadmap-zur-erp-modernisierung-in-der-corona-pandemie-it/

\section{Schlüsselwörter:}

SAP S/4HANA-Migration, Stammdaten, Datenqualität, Migrationsstrategie

\section{Data migration from legacy systems to SAP S/4HANA}

In 2027, the final chord will sound for the manufacturer support of classic SAP ERP solutions - for many users, this could indeed sound in a minor key, because after that there will only be support packages for the former versions - for an additional charge and only for three more years. From 2030 onwards, only SAP S/4HANA will be supported for good. That is why companies that have not yet tackled the changeover to the new technology currently have an acute need to take action. Many have great respect for the complexity of such a migration, especially with regard to master data. However, this is a challenge that companies can master well with careful preparation and the right strategy. This article provides tips for a successful migration.

\section{Keywords:}

data migration, master data, data quality, success, migration strategy 